\title{
POPULATION DYNAMICS AND GENETICS OF PLANT DISEASE: A CASE STUDY OF ANTHER-SMUT DISEASE
}

\author{
Helen Miller Alexander \\ Departments of Botany and Systematics and Ecology, University of Kansas, Lawrence, Kansas 66045 USA \\ Peter H. Thrall and Janis ANTONOvics \\ Department of Botany, Duke University, Durham, North Carolina 27708 USA \\ ANDREW M. JAROSZ \\ Department of Botany and Plant Pathology, Michigan State University, East Lansing, Michigan 48824 USA \\ Peter V. Oudemans ${ }^{2}$ \\ Departments of Botany and Systematics and Ecology, University of Kansas, Lawrence, Kansas 66045 USA
}

\begin{abstract}
A model by Levin and Udovic (1977) emphasizes the need for integration of studies of the numerical abundances and genetic composition of host and pathogen species. We use their conceptual framework to summarize our collaborative research on the ecological genetics of the anther-smut disease of Silene alba caused by the fungus Ustilago violacea. Our theoretical investigations have revealed the importance of the rate and mode of disease transmission on the likelihood of coexistence between host and pathogen. Our empirical studies have quantified patterns of disease spread on local and regional spatial scales and have shown that host genotypes differ greatly in resistance. Comparable genetic variation in pathogen virulence has not yet been demonstrated. The genetic composition of host populations alters numerical dynamics in experimental populations: disease declines in resistant populations, while host and pathogen appear to coexist in susceptible populations. Ecological outcomes also may be affected by the negative relationship between disease incidence and host flowering time, which may constitute a "cost" to resistance. We are currently expanding our work on the ecology and genetics of metapopulation dynamics of host and pathogen.
\end{abstract}

Key words: anther-smut; coexistence; disease ecology; host; pathogen; population ecology; population genetics; Silene alba; transmission; Ustilago violacea.

\section{INTRODUCTION}

The need for an integration of population ecology and population genetics has been repeatedly recognized (Levin and Udovic 1977, Lewontin 1979, Antonovics and Via 1988). Studies of disease demonstrate the necessity of linking numerical and genetic studies. For instance, diseases can have profound effects on numbers of hosts but the outcome of any particular interaction will depend greatly on the genotypes of hosts and pathogens present. In agriculture, crop failures and successes often depend on whether cultivars (analogous to host genotypes) are resistant or susceptible to the pathogens present. Research on plant-pathogen interactions in natural systems has addressed both ecological and genetic questions (Burdon 1987, Augspurger 1988, Parker 1992), but it has proved difficult to over-

\footnotetext{
${ }^{1}$ For reprints of this Special Feature, see footnote 1, p. 989.

${ }^{2}$ Present address: Rutgers Blueberry and Cranberry $\mathrm{Re}$ search Center, Penn State Forest Road, Chatsworth, New Jersey 08019 USA.
}

come the logistical and conceptual difficulties of truly combining research on numbers of organisms with analysis of gene frequencies.

For the last decade, we have struggled with these problems first-hand as we have explored ecological genetic questions with the dioecious host plant Silene alba (white campion, $S$. latifolia) and the fungus $U s$ tilago violacea (anther-smut fungus, Microbotryum violaceum), which infects plants in the Caryophyllaceae (Thrall et al. 1993). These organisms have a fascinating natural history (Baker 1947, Alexander $1990 b$ ): the fungus sporulates in the flowers and in the process prevents normal ovule or pollen production. Insects that visit diseased flowers can deposit spores on healthy plants; flower and bud inoculations yield infected plants (Alexander 1990a, Alexander et al. 1993; S. Altizer, P. Thrall, and J. Antonovics, unpublished data). Infected plants often initially have both healthy and diseased flowers with subsequent systemic spread of the fungus typically leading to host sterili- 
zation. The disease usually has no effect on host survival, although diseased plants had higher mortality rates than healthy plants in years of low overall host mortality (Alexander and Antonovics 1995). Although both plant and pathogen are native to Europe, healthy and diseased plants are common roadside weeds in the Virginia mountains, where our work has been based.

The systemic and sterilizing nature of the pathogen raises many questions of both an ecological and genetic nature. Ecological questions include: can the pathogen regulate the size of the host population? how do host and pathogen coexist? does coexistence occur on the spatial scale of local populations or at the metapopulation level, with extinction or loss of the pathogen occurring in local populations? A geneticist would ask whether there is genetic variation for host resistance or fungal virulence and, if so, whether selection affects frequencies of host or pathogen types. A question that integrates ecology and genetics is whether the genetic composition of host and pathogen populations affects numerical abundances of host and pathogen and their coexistence at either local or regional spatial scales. These and other questions can be addressed using the conceptual framework (Fig. 1) of Levin and Udovic's (1977) coevolution model (= LU) (Antonovics 1992). In this diagram, intraspecific (arrows 1, 3, 5 in Fig. 1) and interspecific (arrows 2, 4, 6 in Fig. 1) densitydependent and frequency-dependent processes can affect the numbers and gene frequencies of the two interacting species. Our goal is to show the importance of the processes in Fig. 1 by summarizing our theoretical and empirical work on the $S$. alba-U. violacea system.

\section{Theoretical ApProaches}

There are three possible numerical outcomes to hostpathogen interactions within a site. At one extreme, the disease could spread to all plants, leading to extinction of the host population as well as the population of the obligate pathogen. In contrast, the pathogen could be lost, resulting in a completely disease-free host population. Finally, host and pathogen may coexist. Central to models of host-pathogen dynamics is the process of disease transmission, including both the mode of disease spread and its rate. For many diseases, the most important factor affecting disease spread is the number of infected individuals, since that determines the total amount of inoculum. Most ecological models of disease incorporate this "density-dependent" transmission (Anderson and May 1981). However, the mode of transmission of anther-smut is different because it is a venereal disease (i.e., an infection transmitted during sexual contact), mediated by pollinators. In such systems, spread of disease should depend on the likelihood of a vector contacting a diseased individual (i.e., fre-

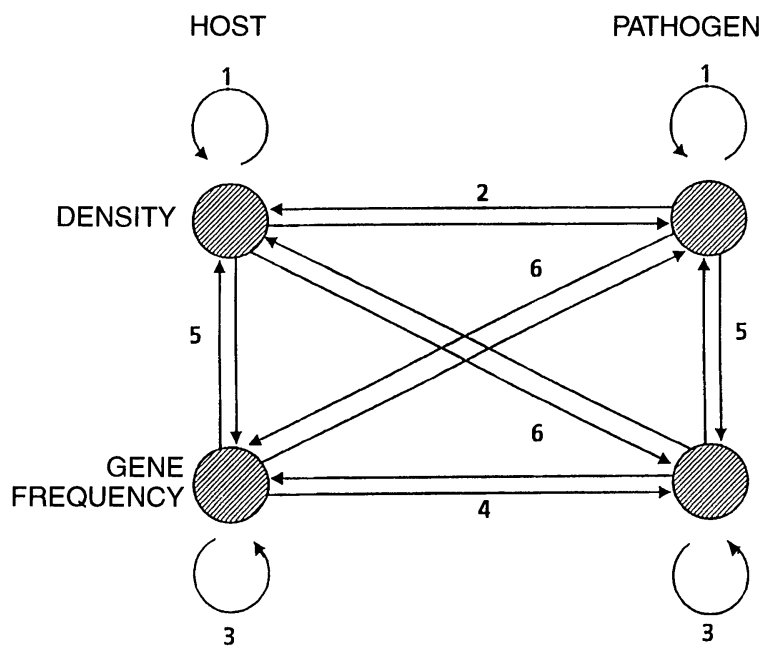

FIG. 1. A conceptual framework for considering numerical and gene frequency changes in two interacting species, based on Levin and Udovic (1977) and Antonovics (1992). Arrows describe intraspecific processes: 1 , intraspecific density dependence; 3 , differential fitness among genotypes unrelated to conspecific density or composition of the other species; and 5, intraspecific density-dependent selection; and interspecific processes: 2 , effect of density of one population on growth rate of the other; 4 , density-independent differential fitness caused by the other species; and 6, density of one species affecting relative fitness of genotypes in the other species, or gene frequency of one species affecting the density of the other. In the text, the combination of an arrow number and a letter refer to intraspecific host $(\mathrm{H})$ or pathogen $(\mathrm{P})$ processes. The combination of an arrow number and two letters refers to interspecific processes, with the order of the letters referring to the direction of the procesess (i.e., $2 \mathrm{HP}$ describes effect of host numbers on pathogen numbers).

quency of disease), as confirmed by our empirical studies (Antonovics and Alexander 1992). Coexistence of host and pathogen is more difficult to achieve in models with frequency-dependent as opposed to density-dependent transmission because coexistence is only possible if host population size is regulated by factors other than the pathogen (Getz and Pickering 1983). Our theoretical work has shown that conditions for coexistence with sexually transmitted diseases are broadened if density-dependent forces are greater for healthy than for diseased plants (Antonovics 1992, 1994, Thrall et al. 1993, 1995). Thus, knowledge of host and pathogen density dependence (arrows $1 \mathrm{H}$ and $1 \mathrm{P}$ in Fig. 1) is necessary to predict interspecific interactions (arrows $2 \mathrm{HP}$ and $2 \mathrm{PH}$ in Fig. 1).

The rate of disease spread also affects the likelihood of coexistence in ecological models (Alexander and Antonovics 1988, Antonovics 1992, 1994, Thrall et al. 1993, Thrall and Jarosz 1994b, Thrall et al. 1995). The resistance and virulence structures of host and pathogen populations could both affect the rate of spread; the focus of our models on venereal diseases has been ge- 
netic variation in resistance. Consider, for example, these equations describing the dynamics of healthy $(X)$ and diseased $(Y)$ plants:

$$
\begin{aligned}
& X_{t+1}=X_{t}\left[1+b-\beta Y_{t} / N_{t}-d\right] \\
& Y_{t+1}=Y_{t}\left[1+\beta X_{t} / N_{t}-d\right]
\end{aligned}
$$

( $b$ is the birth rate, $\beta$ is the disease transmission parameter, and $d$ is the death rate). We can incorporate density-dependent growth most simply by assuming $b$ $=\lambda-\gamma N_{t}$, where $\lambda$ is the maximum host reproductive rate and $\gamma$ is a constant that determines the strength of density dependence. Genetic variation in resistance enters the model as different values of the transmission coefficient, $\beta$, which is the rate at which healthy plants that contact diseased plants become infected (note that nongenetic factors can also affect $\beta$ ). If host populations are monomorphic for $\beta$ values, disease will be lost from resistant (low $\beta$ ) populations and there will either be coexistence or extinction of host and pathogen in susceptible (high $\beta$ ) populations, depending on the rates of host recruitment and mortality (Antonovics 1992, Thrall et al. 1995). Given a fitness cost to resistance, coexistence can occur if populations contain both susceptible and resistant individuals (Antonovics 1992). Surprisingly, polymorphisms can be maintained even if the cost is small; coexistence is easiest to achieve when there is a large difference in susceptibility among the host genotypes (Antonovics and Thrall 1994). Future models must also emphasize stochastic factors, which greatly reduced the likelihood of coexistence in susceptible populations (Thrall and Jarosz 1994b). Overall, our work emphasizes that the host genetic composition can affect the number of diseased individuals (upward diagonal arrow 6HP in Fig. 1).

\section{EMPIRICAL APPROACHES}

\section{Intraspecific interactions}

Given these theoretical insights, what is our empirical knowledge of the various processes depicted in the LU diagram? We first address the numerical dynamics and gene frequency changes that result from intraspecific processes (i.e., arrows 1, 3, 5 in Fig. 1). In natural populations of $S$. alba, host numbers can change dramatically due to the plant's ability to colonize disturbed sites. Evidence consistent with negative host densitydependence (arrow 1H in Fig. 1) comes from long-term surveys of populations (Antonovics et al. 1994), where the rate of plant population increase between years is proportionately less for large as compared to small populations. Actual mechanisms of density dependence are unknown, however, and the result could be explained by other factors such as the age and successional state of the site. We have argued that density dependence is more likely to affect seedlings than adults (Antonovics
1992), as in other short-lived perennials (Shaw and Antonovics 1986). Under this scenario, density dependence would be experienced primarily by healthy plants since diseased seedlings are rare. As noted earlier, this difference in density dependence for diseased and healthy plants increases the likelihood of host-pathogen coexistence in models. Our actual knowledge of seedling dynamics is slim. In retrospect, the fact that the disease can be diagnosed only after flowering has led us to ignore juveniles in the field; thus theoretical work on differential density dependence has opened our eyes to gaps in our empirical knowledge.

In our genetic studies of $S$. alba, we have focused on genetic variation in susceptibility to $U$. violacea. Field experiments (Alexander 1989, Thrall and Jarosz 1994a, Alexander and Antonovics 1995) and greenhouse studies (Alexander et al. 1993) have demonstrated that genotypes or family groups derived from a single population of $S$. alba (Horton's field) differ greatly in their susceptibility to the pathogen $(0-100 \%$ infection, Alexander 1989); this variation is heritable (Alexander and Antonovics 1995). Genetic variation in susceptibility in the field is largely explained by results of inoculation studies (Alexander et al. 1993), suggesting some type of physiological basis to resistance. However, additional predictors of disease levels of genotypes in the field are traits affecting transmission (timing of flowering, flower production) (Alexander et al. 1993, Thrall and Jarosz 1994a), implying genetic differences among plants in exposure to spores. We do not know what factors are responsible for changes in the frequencies of susceptible or resistant types in the absence of the pathogen (arrows $3 \mathrm{H}, 5 \mathrm{H}$ in Fig. 1). Such changes will depend in part on whether resistance has a fitness cost (suggested by Alexander and Antonovics 1995, Biere and Antonovics 1995), thus leading to increases in frequency of susceptible genotypes in the absence of disease. For example, late flowering, which is associated with resistance, leads to a shorter flowering period ( $\mathrm{P}$. Thrall, unpublished data) and there are fewer seeds per fruit $(75 \%$ reduction; Alexander, 1987) for flowers produced in late summer.

Population biology studies of pathogenic fungi have been hampered by the difficulties of identifying individuals in the field. For example, multiple infections of $U$. violacea can occur in a single host plant following artificial inoculation (Day 1980). We currently have no evidence either for or against multiple infections in nature and thus have made the practical decision to consider a diseased plant as a fungal individual. Fungal population size, as measured by the number of diseased plants, often remains relatively constant over time but the disease can also disappear from a site; it does not appear that rapid disease spread has directly led to the demise of host and pathogen populations (Antonovics 
et al. 1994). We lack information on density-dependent regulation in pathogen populations (arrow 1P in Fig. 1 ), however density dependence could operate as a consequence of the defined number of sites for infection (analogous to density dependence of seed germination with limited safe sites).

Our limited studies have revealed no evidence of genetic variation in fungal virulence (Alexander et al. 1993) or in rates of spore production (Alexander and Maltby 1990), however the sample sizes were small. Antonovics et al. (1995) also found no protein electrophoretic polymorphisms (with the exception of a single marker linked to fungal mating type) within or among sites in Virginia. These low levels of variation in $U$. violacea may reflect population bottlenecks occurring with long-distance dispersal of spores from Europe and among geographically distinct sites in North America. Recent work with Randomly Amplified Polymorphic DNA (RAPD) molecular markers of $U$. violacea does reveal variation at the DNA level. Inheritance studies of the RAPD fragments show that they generally behave in Mendelian fashion. Two fungal populations separated by only $3.7 \mathrm{~km}$ had very different allele frequencies at nine loci (P. V. Oudemans, unpublished data). If these loci are neutral, it suggests that there is limited gene flow between sites and that genetic drift may be an important force in determining the genetic composition of fungal populations.

\section{Interspecific interactions}

Actual empirical demonstrations of the effect of disease on host population numbers (arrow 2PH in Fig. 1) are difficult to conduct; a severe effect of a biotic agent on individual host fitness does not necessarily imply an effect on population size if other factors (for example, number of "safe sites") limit plant establishment. Our evidence that the disease can have a negative effect on host numbers comes from yearly surveys of populations, where there was a negative relationship between host population growth rate and disease frequency (Antonovics et al. 1994). In addition, Thrall and Jarosz (1994a) demonstrated that high disease levels reduced rates of seedling recruitment in experimental populations. The complementary question of the effect of host numbers on pathogen numbers (arrow 2HP in Fig. 1) is, on one level, obvious: an obligate pathogen like $U$. violacea is dependent on living hosts. More subtle effects may exist as well. For example, in Eq. 2, the number of healthy plants $(X)$ at a site affects growth of the pathogen population through its effect on transmission.

Missing in the above discussion is the role of genetic variation in numerical dynamics and vice versa. One would expect that the level of disease at a site would affect the frequencies of resistant and susceptible ge- notypes (downward diagonal arrow 6PH in Fig. 1). Although we are currently expanding our genetic surveys, our past work on host resistance variation was largely restricted to one site, Horton's field (Alexander 1989), making it difficult to directly address this question. Of the 20 genotypes collected from this site, 10 were derived from seed from healthy plants while 10 were derived from seed of plants where the disease had spread in only part of the plant (the pathogen is not seed transmitted). The genotypes that had the lowest disease (most resistant) following disease transmission under field conditions (Alexander 1989) had healthy plants as their mothers, which is consistent with the idea that disease is selecting for host resistance. The rapid rates of disease spread in some newly infected populations (Alexander 1990a) as compared to the Horton field site with its long history of disease (Alexander and Antonovics 1988) also could be explained by a higher frequency of resistant genotypes accumulating following exposure to the fungus. Using a field transplant experiment, Elmqvist et al. (1993) further found that $S$. dioica plants derived from a completely healthy site had larger flowers, longer styles, and received more spores than plants derived from a site with high disease, suggesting pathogen selection (mediated by the vector) for floral traits conferring resistance.

If a host population becomes more resistant, rates of transmission should drop; theoretical models suggest that disease is likely to be purged from such populations (Thrall and Jarosz 1994b). Thus, the effect of the pathogen on the genetic composition of host populations (downward diagonal arrow 6PH in Fig. 1) could in turn lead to effects on numbers of pathogens and hosts (upward diagonal arrow 6HP and 2PH in Fig. 1). Empirical observations confirm that disease has disappeared from some natural populations, (Antonovics et al. 1994), but it is difficult to assess the role of genetics. For example, large numbers of healthy and diseased plants were found at the Horton's field site in the early and mid 1980s. More recently, the host population size has declined rapidly at this site, apparently due to successional changes in vegetation. The disease had disappeared at the site by 1991 . The low rates of transmission observed at the site could explain the loss of the pathogen; in fact, simulation models with parameter estimates derived from this site predicted eventual loss of disease (Alexander and Antonovics 1988).

If disease did select for more resistant plant genotypes at Horton's field, the genetic composition of the host population could contribute, through the effects of resistance on transmission, to the decline in pathogen numbers (upward diagonal arrow 6HP in Fig. 1). In an experimental population, transmission rates did decline over time, apparently because the genetic composition of the host population became increasingly more re- 
sistant as susceptible plants were effectively removed by infection (Alexander and Antonovics 1995). Thrall and Jarosz (1994b) studied the numerical consequences of genetic variation in resistance in population cage experiments. Field populations were established with either susceptible or resistant plants. Observations of changes in numbers of healthy and diseased plants over a multiyear period suggested that the disease would eventually be purged in the resistant populations while coexistence of host and pathogen appeared likely in the susceptible populations. These results were qualitatively consistent with computer simulations of frequency-dependent disease transmission models (Thrall and Jarosz 1994b), thus giving us encouragement that our simple models do contain many of the essential features of the system.

\section{Conclusions}

Our theoretical and empirical work on Silene alba and Ustilago violacea illustrate that studies of the population biology of host-pathogen interactions should include analyses of both numerical abundance and genetic composition because these two factors are so intertwined. Clearly, pathogen numbers have the potential to affect the genetic composition of host populations. Changes in the genetic composition of the host population can, in turn, affect host and pathogen numbers. We have begun to extend our theoretical and empirical studies to consider the role of stochastic processes on the numerical dynamics and genetic composition of multiple populations of $S$. alba and $U$. violacea (Thrall and Antonovics 1995). The need for such research is supported both by empirical studies that show small plant populations are more prone to extinction (Antonovics et al. 1994) and by theoretical models that often produce trajectories that pass through very small population sizes (Thrall and Jarosz 1994b, Thrall et al. 1995). For example, even if genetic variation for resistance is present, reduction in population size due to disease may lead to extinction before selection can change the population's genetic composition and allow it to increase in numbers (Gomulkiewicz and Holt 1995). Also, we need to further consider spatial pattern in our models (as in the metapopulation studies; Antonovics et al. 1994, Thrall and Antonovics 1995), given the clearly distance-based process of spore dispersal (Roche et al. 1995).

In our studies, we have striven quite consciously to create a "dialogue" between theoretical and empirical approaches. Our early empirical work on genetic variation in resistance (Alexander 1989) led to the development of theory on the effect of host genetics on hostpathogen coexistence (Antonovics 1992), which in turr inspired further empirical and theoretical studies (Thrall et al. 1993, Thrall and Jarosz 1994a, b). As noted previously, theoretical studies on host-pathogen coexistence also emphasized that empirical data were needed on whether healthy and diseased plants were differentially affected by crowding. Although we initially attempted simulation models of particular populations (Alexander and Antonovics 1988), we now find it more productive to develop models of a general and simple nature. Such models are particularly useful for illustrating what types of processes (for example, frequency vs. density-dependent transmission, densitydependent growth rates) have the largest effects on size or genetic composition of a population. Such a conceptual framework is equally relevant for applied problems. R. C. Lewontin (personal communication) has noted, for example, that ecological questions are difficult to address in schistosomiasis research because dynamical analyses of the entire disease cycle are lacking. Without such analyses, it is hard for the field biologist to know what ecological variables need to be measured and to judge how sensitive the dynamics of the system may be to changes in these variables.

Our simple models have focused on the host and pathogen. It is important to also consider the role of other species such as pollinators/vectors (bees and moths) on the dynamics of $S$. alba and $U$. violacea. For instance, insect visitation can depend on floral traits and some vectors have clear preferences for healthy flowers (Elmqvist et al. 1993, Shykoff and Bucheli 1995; S. Altizer, P. Thrall, and J. Antonovics, unpublished data); vector behavior and disease incidence interact in their effects on disease spread in models (Real et al. 1992). However, consideration of large numbers of interacting species would be unwieldy for either empirical or theoretical study; the ecologist's inherent fascination with complexity can obscure general principles (Harper 1990, May 1990). We also want to avoid the other extreme, where interest in a particular process (i.e., one arrow on the LU figure [Fig. 1]) leads us to neglect all other factors, even though they may influence the outcome of the interactions that intrigue us. Our collaborative work on the ecology and genetics of $S$. alba and $U$. violace $a$ is an attempt to find the middle ground between these extremes.

\section{ACKNOWLEDGMENTS}

This research was supported by NSF grants BSR-8896156 to H. M. Alexander, BSR-8805616 to H. M. Alexander and J. Antonovics, DEB-9119409 to H. M. Alexander and P. V. Oudemans, DEB-9119626 to J. Antonovics, DEB-9207204 to A. M. Jarosz, and BSR-9016343 to P. H. Thrall. University of Virginia's Mountain Lake Biological Station provided postdoctoral fellowships for $\mathrm{H}$. M. Alexander and logistical support for many studies. We especially thank the field and laboratory assistants who contributed to this research.

$$
\text { Literature Cited }
$$

Alexander, H. M. 1987. Pollination limitation in a population of Silene alba infected by the anther-smut fungus, Ustilago violacea. Journal of Ecology 75:771-780. 
$\longrightarrow \rightarrow$-. 1989. An experimental field study of anther-smut disease of Silene alba caused by Ustilago violacea: genotypic variation and disease incidence. Evolution 43:835847.

$\rightarrow-$ 1990a. Epidemiology of anther-smut infection of Silene alba caused by Ustilago violacea: patterns of spore deposition and disease incidence. Journal of Ecology 78: 166-179.

1990b. Dynamics of plant-pathogen interactions in natural plant communities. Pages 31-45 in J. J. Burdon and S. R. Leather, editors. Pests, pathogens, and plant communities. Blackwell Scientific, Oxford, England

$\rightarrow$ Alexander, H. M., and J. Antonovics. 1988. Disease spread and population dynamics of anther-smut infection of Silene alba caused by the fungus Ustilago violacea. Journal o $\rightarrow$ Ecology 76:91-104.

$\rightarrow$ Alexander, H. M., and J. Antonovics. 1995. Spread of anthersmut disease (Ustilago violacea) and character correlations in a genetically variable experimental population of Silen alba. Journal of Ecology 83:783-794.

$\rightarrow$ Alexander, H. M., J. Antonovics, and A. W. Kelly. 1993. Genotypic variation in plant disease resistance: physiolog ical resistance in relation to field disease transmission. Journal of Ecology 81:325-334.

$\rightarrow$ Alexander, H. M., and A. Maltby. 1990. Anther-smut infection of Silene alba caused by Ustilago violacea: factors determining fungal reproduction. Oecologia 84:249-253.

$\rightarrow$ Anderson, R. M., and R. M. May. 1981. The population dynamics of microparasites and their invertebrate hosts Philosophical Transactions of the Royal Society of London B 292:451-524.

Antonovics, J. 1992. Toward community genetics. Pages 426-449 in R. S. Fritz and E. L. Simms, editors. Plant resistance to herbivores and pathogens. University of Chicago Press, Chicago, Illinois, USA.

- 1994. The interplay of numerical and gene-frequency dynamics in host-pathogen systems. Pages 129-145 in L. A. Real, editor. Ecological genetics. Princeton University Press, Princeton, New Jersey, USA.

$\rightarrow$ Antonovics, J., and H. M. Alexander. 1992. Epidemiology of anther-smut infection of Silene alba $(=S$. latifolia $)$ caused by Ustilago violacea: patterns of spore deposition in experimental populations. Proceedings of the Royal Society of London B 250:157-163.

$\rightarrow$ Antonovics, J., Y. Iwasa, and M. P. Hassell. 1995. A generalized model of parasitoid, venereal, and vector-based transmission processes. American Naturalist 145:661-675.

Antonovics, J., D. Stratton, P. H. Thrall, and A. Jarosz. 1995. Anther-smut disease (Ustilago violacea) of fire pink (Silene virginica): its biology and relationship to the anther-smut disease of white campion. American Midland Naturalist in press.

$\rightarrow$ Antonovics, J., and P. H. Thrall. 1994. The cost of resistance and the maintenance of genetic polymorphism in host pathogen systems. Proceedings of the Royal Society of London B 257:105-110.

Antonovics, J., P. H. Thrall, A. M. Jarosz, and D. Stratton. 1994. Ecological genetics of metapopulations: the Silene Ustilago plant-pathogen system. Pages 146-170 in L. A Real, editor. Ecological genetics. Princeton University Press, Princeton, New Jersey, USA.

Antonovics, J., and S. Via. 1988. Genetic influences on the distribution and abundance of plants. Pages 185-203 in A. J. Davy, M. J. Hutchings, and A. R. Watkinson, editors. Plant population ecology. Blackwell Scientific, Oxford, England.

Augspurger, C. K. 1988. Impact of pathogens on natural plant populations. Pages 413-433 in A. J. Davy, M. J. Hutchings, and A. R. Watkinson, editors. Plant population ecology. Blackwell Scientific, Oxford, England.

Baker, H. G. 1947. Infection of species of Melandrium by Ustilago violacea (Pers.) Fuckel and the transmission of the resultant disease. Annals of Botany 11:333-348.

Biere, A., and J. Antonovics. 1995. Sex-specific costs of resistance to the fungal pathogen Ustilago violacea ( $\mathrm{Mi}$ crobotryum violaceum) in Silene alba. Evolution, in press.

Burdon, J. J. 1987. Diseases and plant population biology. Cambridge University Press. Cambridge, England.

Day, A. W. 1980. Competition and distribution studies of genetically marked strains of Ustilago violacea in the same host plant. Botanical Gazette 141:313-320.

Elmqvist, T., D. Liu, U. Carlsson, and B. E. Giles. 1993. Anther-smut infection in Silene dioica: variation in floral morphology and patterns of spore deposition. Oikos 68: 207-216.

Getz, W. M., and J. Pickering. 1983. Epidemic models: thresholds and population regulation. American Naturalist 121:892-898.

Gomulkiewicz, R., and R. D. Holt. 1995. When does evolution by natural selection prevent extinction? Evolution 49:201-207.

Harper, J. L. 1990. Pests, pathogens, and plant communities: an introduction. Pages 3-14 in J. J. Burdon and S. R. Leather, editors. Pests, pathogens, and plant communities. Blackwell Scientific, Oxford, England.

Levin, S. A., and J. D. Udovic. 1977. A mathematical model of coevolving populations. American Naturalist 111:657675 .

Lewontin, R. C. 1979. Fitness, survival, and optimality. Pages 3-21 in D. H. Horn, R. Mitchell, and G. R. Stairs, editors. Analysis of ecological systems. Ohio State University Press. Columbia, Ohio, USA.

May, R. M. 1990. Population biology and population genetics of plant-pathogen associations. Pages 309-325 in J. J. Burdon and S. R. Leather, editors. Pests, pathogens, and plant communities. Blackwell Scientific, Oxford, England.

Parker, M. A. 1992. Disease and plant population genetic structure. Pages 345-362 in R. S. Fritz and E. L. Simms, editors. Plant resistance to herbivores and pathogens. University of Chicago Press, Chicago, Illinois, USA.

Real, L. A., E. A. Marschall, and B. M. Roche. 1992. Individual behavior and pollination ecology: implications for the spread of sexually transmitted plant diseases. Pages 492-508 in D. DeAngelis and L. Gross, editors. Populations, communities, and ecosystems: a perspective from modeling at the level of individual organisms. Chapman and Hall, New York, New York, USA.

Roche, B. M., H. M. Alexander, and A. D. Maltby. 1995. Dispersal and disease gradients of anther-smut infection of Silene alba at different life stages. Ecology 76:1863-1871.

Shaw, R. G., and J. Antonovics. 1986. Density-dependence in Salvia lyrata, a herbaceous perenniall: the effects of experimental alteration of seed densities. Journal of Ecology 74:797-813

Shykoff, J. A., and E. Bucheli. 1995. Pollinator visitation patterns, floral rewards and the probability of transmission of Microbotryum violaceum, a venereal disease of plants. Journal of Ecology 83:189-198.

Thrall, P. H., and J. Antonovics. 1995. Theoretical and empirical studies of metapopulations: population and genetic dynamics of the Silene-Ustilago system. Canadian Journal of Botany 73 (Supplement 1):S1249-S1258.

$\rightarrow$ Thrall, P. H., J. Antonovics, and D. W. Hall. 1993. Host and pathogen coexistence in sexually transmitted and vector- 
borne diseases characterized by frequency-dependent dis $\rightarrow$ Thrall, P. H., and A. M. Jarosz. 1994a. Host-pathogen dyease transmission. American Naturalist 142:543-552. namics in experimental populations of Silene alba and Ustilago violacea I. Ecological and genetic determinants of disease spread. Journal of Ecology 82:549-559.

$\rightarrow$ Thrall, P. H., A. Biere, and J. Antonovics. 1993. Plant lifehistory and disease susceptibility: the occurrence of Ustilago violacea on different species within the Caryophyl laceae. Journal of Ecology 81:489-498.

$\rightarrow$ Thrall, P. H., A. Biere, and M. K. Uyenoyama. 1995. Frequency dependent disease transmission and the dynamics of the Silene-Ustilago host/pathogen system. American namics in experimental populations of Silene alba and Ustilago violacea. II. Experimental tests of theoretical models. Journal of Ecology 82:561-570. 